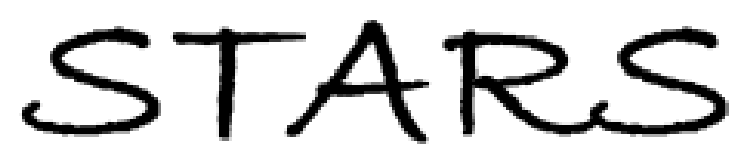

University of Central Florida

STARS

$1-1-2009$

\title{
Modal, spectral, and polarization entanglement in guided-wave parametric down-conversion
}

Mohammed F. Saleh

Bahaa E. A. Saleh

University of Central Florida

Malvin Carl Teich

Find similar works at: https://stars.library.ucf.edu/facultybib2000

University of Central Florida Libraries http://library.ucf.edu

This Article is brought to you for free and open access by the Faculty Bibliography at STARS. It has been accepted for inclusion in Faculty Bibliography 2000 s by an authorized administrator of STARS. For more information, please contact STARS@ucf.edu.

\section{Recommended Citation}

Saleh, Mohammed F.; Saleh, Bahaa E. A.; and Teich, Malvin Carl, "Modal, spectral, and polarization entanglement in guided-wave parametric down-conversion" (2009). Faculty Bibliography 2000s. 2086. https://stars.library.ucf.edu/facultybib2000/2086

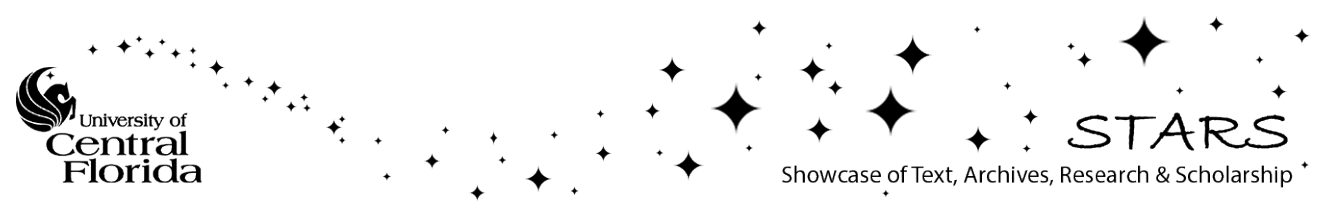




\title{
Modal, spectral, and polarization entanglement in guided-wave parametric down-conversion
}

\author{
Mohammed F. Saleh* \\ Department of Electrical and Computer Engineering, Quantum Photonics Laboratory, Boston University, \\ Boston, Massachusetts 02215, USA \\ Bahaa E. A. Saleh \\ Department of Electrical and Computer Engineering, Quantum Photonics Laboratory, Boston University, \\ Boston, Massachusetts 02215, USA \\ and Quantum Photonics Laboratory, College of Optics and Photonics (CREOL), University of Central Florida, \\ Orlando, Florida 32816, USA \\ Malvin Carl Teich \\ Department of Electrical and Computer Engineering, Quantum Photonics Laboratory, Boston University, \\ Boston, Massachusetts 02215, USA \\ and Department of Physics, Boston University, Boston, Massachusetts 02215, USA
}

(Received 20 February 2009; published 21 May 2009)

\begin{abstract}
We examine the modal, spectral, and polarization entanglement properties of photon pairs generated in a nonlinear periodically poled two-mode waveguide (one-dimensional planar or two-dimensional circular) via nondegenerate spontaneous parametric down-conversion. Any of the possible degrees of freedom-mode number, frequency, or polarization — can be used to distinguish the down-converted photons while the others serve as attributes of entanglement. Distinguishing the down-converted photons based on their mode numbers enables us to efficiently generate spectral or polarization entanglement that is either narrowband or broadband. On the other hand, when the generated photons are distinguished by their frequencies in a type- 0 process, modal entanglement turns out to be an efficient alternative to polarization entanglement. Moreover, modal entanglement in type-II down-conversion may be used to generate a doubly entangled state in frequency and polarization.
\end{abstract}

DOI: 10.1103/PhysRevA.79.053842

PACS number(s): 42.65.Wi, 42.65.Lm, 42.50.Dv

\section{INTRODUCTION}

Photon pairs generated via the nonlinear process of spontaneous parametric down-conversion (SPDC) can exhibit entanglement in multiple degrees of freedom: spectral, spatial, and polarization [1]. Entanglement arises from the multiple possibilities for satisfying energy and momentum conservation, as required by the parametric interaction process. Spectral entanglement is exhibited equivalently in time or energy [2]; spatial entanglement may be manifested as entanglement in wave-vector direction [3], transverse direction [4], orbitalangular-momentum (OAM) [5], or spatial parity [6,7]. A proper description of the quantum state of the photon pair (biphoton) requires a Hilbert space that includes all of the pertinent degrees of freedom. One of these degrees of freedom may be arbitrarily chosen to distinguish the two photons, while the other two are used to describe the state. The term hyperentanglement has been used to describe states defined over multiple degrees of freedom [8].

While the polarization variable is intrinsically binary, spectral and spatial variables are generally continuous. Nevertheless, binary spectral or spatial degrees of freedom can be extracted by selection of a subspace of dimension two (a qubit). Examples are frequency selection by use of narrow spectral filters [9], direction selection by use of pinholes in

\footnotetext{
*mohsaleh@bu.edu
}

the far field [10], and lower-order OAM-mode selection by suppression of higher-order modes [11]. Another approach to binarization is based on constraining the parametric process in such a way that only two values of the continuous variable are permitted; such configurations include narrowband spectral entanglement [12] and type-II noncollinear downconversion in which only two directions (as determined by intersecting rings) are permitted [13]. A third approach is to map the larger Hilbert space onto a binary space, as in spatial-parity entanglement $[6,14]$.

In this paper, we consider the generation of biphotons by means of SPDC in a nonlinear waveguide. This configuration confines the photons to a single direction of propagation and discretizes the spatial degree of freedom to the waveguide spatial modes. For a two-mode waveguide, the spatial degree of freedom is binary, representing a modal qubit. The waveguide also supports two polarizations [e.g., TE and TM in a one-dimensional (1D) geometry]. Moreover, the spectral distributions of the down-converted photons may be constrained to yield a pair of narrow spectral lines defining a spectral qubit, or alternatively, may be made more flexible so as to generate photon pairs with broad spectral entanglement; these conditions are achieved by making use of periodic and linearly chirped poling of the nonlinear medium, respectively.

In addition to the advantage of spatial binarization, the two-mode waveguide has the merit of combining a higher rate of biphoton generation, normally obtained in collineardegenerate bulk SPDC, with the photon separability gener- 
ally offered by a noncollinear configuration. Moreover, processing of the generated biphotons for applications in quantum information [15] is often facilitated by the use of guided-wave devices.

In this paper, we offer a comprehensive theoretical study of the properties and applications of modal, spectral, and polarization entanglement of biphotons generated via SPDC in $1 \mathrm{D}$ planar and two-dimensional (2D) circular waveguides. In particular, we consider biphotons generated via different types of interactions and with the use of a continuous-wave (cw) pump. Prior work in this area has been limited and has made use of a pulsed, rather than cw, pump [16,17].

The paper is organized as follows. In Sec. II, we develop a general theory for SPDC in periodically poled multimode waveguides. In Sec. III, we determine the waveguide parameters required to generate modal and spectral or polarization entanglement in 1D planar waveguides using either type- 0 or type-II interactions. A similar study is carried out in Sec. IV for 2D circular waveguides (optical fibers). Features and applications of modal entanglement are considered in Sec. V. Finally, the conclusions are presented in Sec. VI

\section{SPONTANEOUS PARAMETRIC DOWN-CONVERSION IN MULTIMODE WAVEGUIDES}

Consider an SPDC process in a multimode waveguide, where a pump wave $p$, is down-converted into a signal wave $s$ and an idler wave $i$. Using time-dependent perturbation theory, the two-photon state $|\Psi\rangle$ can be written as [12]

$$
|\Psi\rangle \sim \iint d \mathbf{r} d t \mathbf{d}(\mathbf{r}) \mathbf{E}_{p}(\mathbf{r}, t) \hat{\mathbf{E}}_{s}^{(-)}(\mathbf{r}, t) \hat{\mathbf{E}}_{i}^{(-)}(\mathbf{r}, t)|0,0\rangle,
$$

where $\mathbf{d}(\mathbf{r})$ is the second-order nonlinear-coefficient tensor; $\mathbf{E}_{p}$ is the pump electric field at position $\mathbf{r}$ and time $t$, treated classically and assumed to be undepleted; $\hat{\mathbf{E}}_{q}^{(-)}$are the negative-frequency parts of the signal and idler electric field operators $(q=s, i)$ at position $\mathbf{r}$ and time $t$; and $|0,0\rangle$ is the vacuum state of the signal and the idler.

For guided waves propagating along the $x$ direction, the electric field operator of the signal and idler is written as

$$
\begin{aligned}
\hat{\mathbf{E}}_{q}^{(-)}(\mathbf{r}, t)= & \int d \omega_{q} \sum_{m_{q}, \sigma_{q}} \hat{a}_{m_{q}, \sigma_{q}}^{\dagger}\left(\omega_{q}\right) u_{m_{q}, \sigma_{q}}\left(\omega_{q}, y, z\right) \\
& \times \exp \left[j \omega_{q} t-j \beta_{m_{q}, \sigma_{q}}\left(\omega_{q}\right) x\right],
\end{aligned}
$$

where $\omega_{q}$ is the angular frequency; $\sigma_{q}$ and $m_{q}$ are the polarization and spatial-mode indexes, respectively; $\beta_{m_{q}, \sigma_{q}}$ is the propagation constant; $u_{m_{q}, \sigma_{q}}$ is the transverse-field profile in the $y-z$ plane; and $\hat{a}_{m_{q}, \sigma_{q}}^{\dagger}$ is the creation operator for the wave $q$. For the classical pump electric field, a similar expression can be obtained by taking the complex conjugate of Eq. (2) then replacing the creation operator with the wave complex amplitude.

Substituting Eq. (2) into Eq. (1), assuming that d(r) depends only on $x$, and noting that the integration over $t$ yields the delta function $\delta\left(\omega_{p}-\omega_{s}-\omega_{i}\right)$, the two-photon state becomes

$$
|\Psi\rangle \sim \int d \omega_{s} \sum_{\mathbf{m}, \boldsymbol{\sigma}} \Phi_{\mathbf{m}, \boldsymbol{\sigma}}\left(\omega_{s}\right)\left|\omega_{s}, m_{s}, \sigma_{s}\right\rangle\left|\omega_{i}, m_{i}, \sigma_{i}\right\rangle,
$$

where

$$
\begin{gathered}
\Phi_{\mathbf{m}, \boldsymbol{\sigma}}\left(\omega_{s}\right)=A_{\mathbf{m}, \boldsymbol{\sigma}}\left(\omega_{s}\right) \int d x \mathbf{d}(x) \exp \left[j \Delta \beta_{\mathbf{m}, \boldsymbol{\sigma}}\left(\omega_{s}\right) x\right], \\
A_{\mathbf{m}, \boldsymbol{\sigma}}\left(\omega_{s}\right)=\iint d y d z \prod_{q=p, s, i} u_{m_{q}, \sigma_{q}}\left(\omega_{q}, y, z\right),
\end{gathered}
$$

$\omega_{i}=\omega_{p}-\omega_{s}, \quad \mathbf{m}=\left(m_{s}, m_{i}\right), \quad \boldsymbol{\sigma}=\left(\sigma_{s}, \sigma_{i}\right), \quad$ and $\quad \Delta \beta_{\mathbf{m}, \boldsymbol{\sigma}}\left(\omega_{s}\right)$ $=\beta_{m_{p}, \sigma_{p}}\left(\omega_{p}\right)-\beta_{m_{s}, \sigma_{s}}\left(\omega_{s}\right)-\beta_{m_{i}, \sigma_{i}}\left(\omega_{i}\right)$ is the phase mismatch. The factor $A_{\mathbf{m}, \boldsymbol{\sigma}}$ represents the spatial overlap of the transverse profiles of the interacting modes. The square magnitude of $\Phi_{\mathbf{m}, \boldsymbol{\sigma}}\left(\omega_{s}\right)$ represents the SPDC spectrum when the signal and the idler mode numbers are $\mathbf{m}=\left(m_{s}, m_{i}\right)$ and their polarization indexes are $\boldsymbol{\sigma}=\left(\sigma_{s}, \sigma_{i}\right)$.

Since the generated photons are collinear, their directions cannot be used to distinguish the two photons. If the mode number is used in this capacity, then the two-photon state described in Eq. (3) may be written as

$$
|\Psi\rangle \sim \int d \omega_{s} \sum_{\mathbf{m}, \boldsymbol{\sigma}} \Phi_{\mathbf{m}, \boldsymbol{\sigma}}\left(\omega_{s}\right)\left|\omega_{s}, \sigma_{s}\right\rangle_{m_{s}}\left|\omega_{i}, \sigma_{i}\right\rangle_{m_{i}} .
$$

Alternatively, using frequency as the identifier, the twophoton state takes the form

$$
|\Psi\rangle \sim \int d \omega_{s} \sum_{\mathbf{m}, \boldsymbol{\sigma}} \Phi_{\mathbf{m}, \boldsymbol{\sigma}}\left(\omega_{s}\right)\left|m_{s}, \sigma_{s}\right\rangle_{\omega_{s}}\left|m_{i}, \sigma_{i}\right\rangle_{\omega_{i}} .
$$

We may also use polarization as the identifier and write a similar expression for the two-photon state. These expressions for the state are, of course, equivalent.

We now derive expressions for the spectral function $\Phi_{\mathbf{m}, \boldsymbol{\sigma}}$ for quasi-phase-matched (QPM) structures. Such structures are designed to phase match a specific type of interaction (type-0 or I or II) via a certain component of the secondorder nonlinear tensor; hence, $\mathbf{d}$ can be replaced by its effective value $d_{\text {eff. }}$. Using electric poling, the nonlinear coefficient is made to alternate between $\pm d_{\text {eff }}$ along one of the crystal principle axes, say $x$. The poling period can be either uniform or variable. We consider these two cases in turn.

Uniform Poling. For uniform poling with period $\Lambda_{0}$, the spatial distribution of the nonlinear coefficient can be represented as a sum of distinct Fourier components, $d_{\mathrm{eff}}(x)$ $=\sum_{\kappa=1}^{\infty} \tilde{d}_{\kappa} \exp \left[-j \int_{0}^{x} d x K_{\kappa}(x)\right]$, where $\tilde{d}_{\kappa}=(2 / \pi \kappa)\left|d_{\mathrm{eff}}\right|$ and $K_{\kappa}(x)=\left(2 \pi \kappa / \Lambda_{0}\right)$ represent the amplitude and spatial frequency of the $\kappa$ th Fourier component, respectively. Only that Fourier component whose phase is close to, or equal to, the phase mismatch $\Delta \beta_{\mathbf{m}, \boldsymbol{\sigma}}\left(\omega_{s}\right)$ will contribute to $\Phi_{\mathbf{m}, \boldsymbol{\sigma}}\left(\omega_{s}\right)$. Carrying out the integration in Eq. (4) for a waveguide of length $L$, we obtain

$$
\begin{aligned}
\Phi_{\mathbf{m}, \boldsymbol{\sigma}}\left(\omega_{s}\right)= & A_{\mathbf{m}, \boldsymbol{\sigma}}^{\prime}\left(\omega_{s}\right) \operatorname{sinc}\left[\Delta \widetilde{\beta}_{\mathbf{m}, \boldsymbol{\sigma}}\left(\omega_{s}\right) L / 2 \pi\right] \\
& \times \exp \left[j \Delta \widetilde{\beta}_{\mathbf{m}, \boldsymbol{\sigma}}\left(\omega_{s}\right) L / 2\right],
\end{aligned}
$$

where 


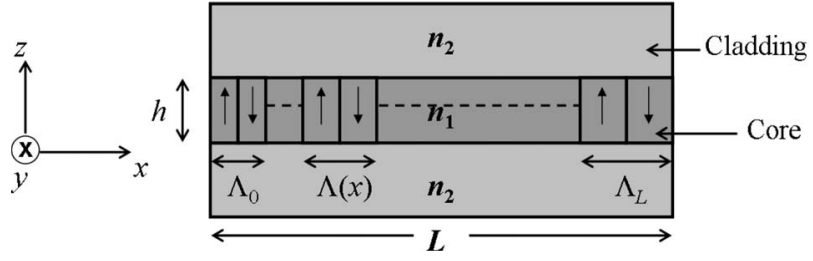

FIG. 1. Sketch of a 1D planar waveguide. The waveguide is a slab of dielectric medium of thickness $h$ with a uniform refractive index $n_{1}$ surrounded by media of lower refractive index $n_{2}$. The inner medium and the outer media are known as the core and cladding, respectively. The nonlinear coefficient of the core is poled with a period $\Lambda(x)$; arrows indicate the poling direction. The poling periods at $x=0$ and $x=L$ are denoted $\Lambda_{0}$ and $\Lambda_{L}$, respectively.

$$
\Delta \widetilde{\beta}_{\mathbf{m}, \boldsymbol{\sigma}}\left(\omega_{s}\right)=\Delta \beta_{\mathbf{m}, \boldsymbol{\sigma}}\left(\omega_{s}\right)-\frac{2 \pi \kappa}{\Lambda_{0}},
$$

$A_{\mathbf{m}, \boldsymbol{\sigma}}^{\prime}\left(\omega_{s}\right)=(2 L / \pi \kappa)\left|d_{\mathrm{eff}}\right| A_{\mathbf{m}, \boldsymbol{\sigma}}\left(\omega_{s}\right), \quad$ and $\quad \operatorname{sinc}(\theta)=\sin (\pi \theta) /$ $(\pi \theta)$. From a practical perspective, the poling period is determined by satisfying the quasi-phase-matching condition at a certain frequency $\omega_{s}$, i.e., $\Delta \widetilde{\beta}_{\mathbf{m}, \boldsymbol{\sigma}}\left(\omega_{s}\right)=0$, or $\Delta \beta_{\mathbf{m}, \boldsymbol{\sigma}}\left(\omega_{s}\right)$ $=2 \pi \kappa / \Lambda_{0}$.

Linearly Chirped Poling. For linearly chirped poling with a slowly varying spatial frequency, we can still make use of a Fourier series to represent the spatial distribution of the nonlinear coefficient with $K_{\kappa}(x)=2 \pi \kappa / \Lambda(x)=2 \pi \kappa / \Lambda_{0}-\zeta x$, where $\zeta$ is the chirp parameter [18]. It is clear that it is the spatial frequency, rather than the spatial period, that is chirped. In this case, Eq. (4) yields

$$
\Phi_{\mathbf{m}, \boldsymbol{\sigma}}\left(\omega_{s}\right)=A_{\mathbf{m}, \boldsymbol{\sigma}}^{\prime \prime}\left(\omega_{s}\right) \exp \left[\frac{-j \Delta \widetilde{\beta}_{\mathbf{m}, \boldsymbol{\sigma}}^{2}\left(\omega_{s}\right)}{2 \zeta}\right][\gamma(L)-\gamma(0)],
$$

where $\quad A_{\mathbf{m}, \boldsymbol{\sigma}}^{\prime \prime}\left(\omega_{s}\right)=\left|d_{\mathrm{eff}}\right| A_{\mathbf{m}, \boldsymbol{\sigma}}\left(\omega_{s}\right) \sqrt{2 j / \pi \zeta \kappa^{2}}, \quad \gamma(x)=$ erfi $\times\left\{\left[\Delta \widetilde{\beta}_{\mathbf{m}, \boldsymbol{\sigma}}\left(\omega_{s}\right)+\zeta x\right] / \sqrt{-2 j \zeta}\right\}, \operatorname{erfi}(\theta)=-j \operatorname{erf}(j \theta)$ and $\operatorname{erf}(\cdot)$ is the error function. In the remainder of this paper, we use $\kappa$ $=1$ to compute the poling periods since this results in the strongest nonlinear interaction and poling periods are not limited by fabrication techniques.

\section{MODAL ENTANGLEMENT IN 1D PLANAR WAVEGUIDES}

We now consider the generation of entangled photons via nondegenerate SPDC in a 1D planar waveguide. The structure comprises a dielectric slab of thickness $h$ and refractive index $n_{1}$ embedded in dielectric media with lower refractive index $n_{2}$, as illustrated in Fig. 1. The transverse profile of mode $m_{q}$ inside the slab is [19]

$$
u_{m_{q}, \sigma_{q}}\left(\omega_{q}, y, z\right) \propto \begin{cases}\cos \left[k_{m_{q}}^{(z)}, \sigma_{q}\left(\omega_{q}\right) z\right], & m_{q}=\text { even } \\ \sin \left[k_{m_{q}, \sigma_{q}}^{(z)}\left(\omega_{q}\right) z\right], & m_{q}=\text { odd },\end{cases}
$$

while outside the slab it is

$$
u_{m_{q}, \sigma_{q}}\left(\omega_{q}, y, z\right) \propto \exp \left[-\gamma_{m_{q}, \sigma_{q}}\left(\omega_{q}\right) z\right],
$$

where $k_{m_{q}, \sigma_{q}}^{(z)}=\left(k_{m_{q}, \sigma_{q}}^{2}-\beta_{m_{q}, \sigma_{q}}^{2}\right)^{1 / 2}$ is the $z$ component of the wave vector $k_{m_{q}, \sigma_{q}}=n_{1} \omega_{q} / c, \quad \gamma_{m_{q}, \sigma_{q}}=\left(\beta_{m_{q}, \sigma_{q}}^{2}-\widetilde{k}_{m_{q}, \sigma_{q}}^{2}\right)^{1 / 2}$, $\tilde{k}_{m_{q}, \sigma_{q}}=n_{2} \omega_{q} / c$, and $c$ is the velocity of light in free space. Note that both $n_{1}$ and $n_{2}$ are frequency dependent.

Within the slab, the mode transverse profiles are either even or odd functions. The propagation constants of the modes can be determined using the dispersion relation for the planar waveguide. For the TE wave ( $o$ polarization),

$$
\tan ^{2}\left[\frac{h}{2} \sqrt{k_{m_{q}, \sigma_{q}}^{2}-\beta_{m_{q}, \sigma_{q}}^{2}}-\frac{m_{q} \pi}{2}\right]=\frac{\beta_{m_{q}, \sigma_{q}}^{2}-k_{m_{q}, \sigma_{q}}^{2}}{\widetilde{k}_{m_{q}, \sigma_{q}}^{2}-\beta_{m_{q}, \sigma_{q}}^{2}},
$$

whereas for the TM wave (e polarization), the right-hand side (RHS) of the above equation must be multiplied by $n_{1}^{2} / n_{2}^{2}$

Substituting Eq. (11) into Eqs. (4) and (5), we can determine the functions $\Phi_{\mathbf{m}, \boldsymbol{\sigma}}\left(\omega_{s}\right)$, which in turn determine the quantum state set forth in Eq. (3). We consider a two-mode waveguide for which the fundamental mode $\left(m_{q}=0\right)$ is even and the next mode $\left(m_{q}=1\right)$ is odd. If the down-converted photons have different spatial parity, the pump mode must be odd so that the spatial overlap integral in Eq. (5), which determines $A_{\mathbf{m}, \boldsymbol{\sigma}}\left(\omega_{s}\right)$ and hence $\Phi_{\mathbf{m}, \boldsymbol{\sigma}}\left(\omega_{s}\right)$, does not vanish.

We seek to generate an entangled state of the form

$$
\begin{aligned}
|\Psi\rangle \sim & \int \mathrm{d} \omega_{s}\left[\Phi_{0,1, \boldsymbol{\sigma}}\left(\omega_{s}\right)\left|\omega_{s}, 0, \sigma_{s}\right\rangle\left|\omega_{i}, 1, \sigma_{i}\right\rangle+\Phi_{1,0, \boldsymbol{\sigma}}\left(\omega_{s}\right)\right. \\
& \left.\times\left|\omega_{s}, 1, \sigma_{s}\right\rangle\left|\omega_{i}, 0, \sigma_{i}\right\rangle\right],
\end{aligned}
$$

i.e., if the signal is in the even mode, the idler must be in the odd mode, and vice versa. This requires that the phasematching condition be satisfied for each of these two possibilities. For a fixed geometry and material parameters, we find a single poling period $\Lambda_{0}$ of the nonlinear coefficient at which these conditions, $\Delta \beta_{0,1, \boldsymbol{\sigma}}\left(\omega_{s}\right)=2 \pi / \Lambda_{0}$ and $\Delta \beta_{1,0, \boldsymbol{\sigma}}\left(\omega_{s}\right)=2 \pi / \Lambda_{0}$, are simultaneously met. We achieve this by plotting the poling period $\Lambda_{0}$ as a function of the signal frequency $\omega_{s}$, or as a function of the signal wavelength $\lambda_{s}$, for each of these two conditions, and search for intersections. The example shown in Fig. 2 reveals that this may occur at a single frequency or, when the two curves are tangential, over a broad spectral band. Once the poling period is selected and fixed, we determine if the spectral functions $\Phi_{0,1, \boldsymbol{\sigma}}\left(\omega_{s}\right)$ and $\Phi_{1,0, \boldsymbol{\sigma}}\left(\omega_{s}\right)$ overlap, i.e., have some common spectral band. If they do, then the two-photon state exhibits entanglement.

Example. A laser source with wavelength $\lambda_{p}=532 \mathrm{~nm}$ and in mode $m_{p}=1$ is used to pump a $1 \mathrm{D}$ periodically poled KTP waveguide with fractional refractive-index change $\Delta \approx 1-n_{2} / n_{1}=0.05$. The core refractive indexes along the $y$ and $z$ axes are given by the KTP Sellmeier equations [20],

$$
n_{1,(y)}^{2}(\lambda)=3.45018+\frac{0.04341}{\lambda^{2}-0.04597}+\frac{16.98825}{\lambda^{2}-39.43799},
$$



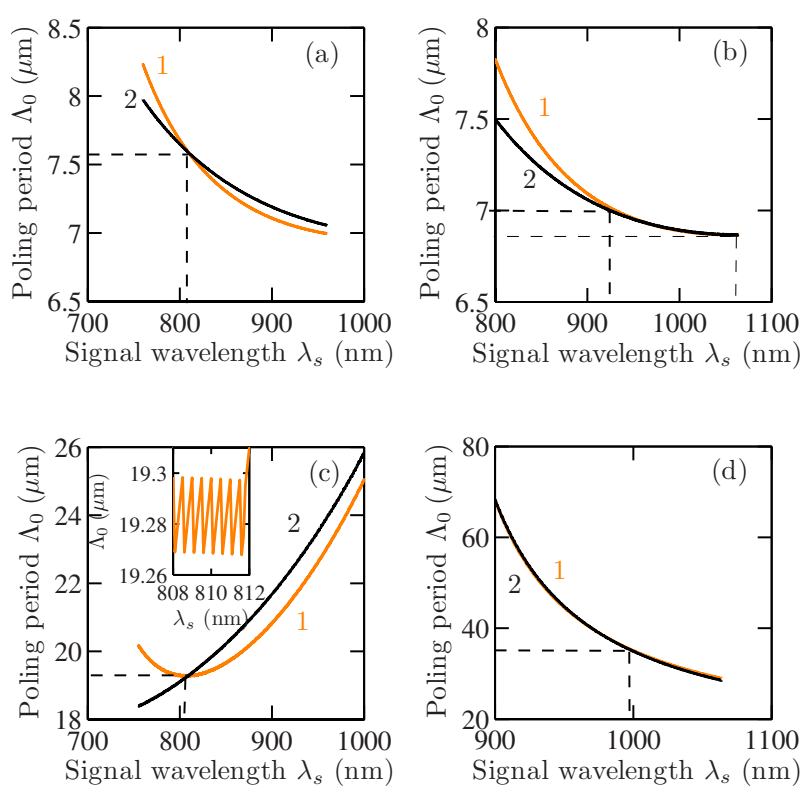

FIG. 2. (Color online) Values of the poling period $\Lambda_{0}$ required to satisfy quasi-phase-matching as a function of the signal wavelength $\lambda_{s}$, for four values of the waveguide thickness $h$. Calculations are presented for a KTP planar waveguide with $\Delta=0.05$ pumped by a laser whose wavelength is $\lambda_{p}=532 \mathrm{~nm}$ and whose modal structure coincides with $m_{p}=1$. The core refractive indexes are determined using the Sellmeier equations for KTP. The orange (black) curves represent condition 1 (2), namely, that the signal (idler) mode number $m_{s}\left(m_{i}\right)$ is 0 and that the idler (signal) mode number $m_{i}\left(m_{s}\right)$ is 1 . Dashed lines indicate intersection points or ranges. (a) Type- 0 $(e, e, e)$ with waveguide thickness $h=1.625 \mu \mathrm{m}$. (b) Type-0 $(e, e, e)$ with $h=1.49 \mu \mathrm{m}$. (c) Type-II $(o, e, o)$ with $h=1.617 \mu \mathrm{m}$. The inset shows the behavior of the poling period for condition 1 near the intersection point. (d) Type-II $(e, o, o)$ with $h=1.56 \mu \mathrm{m}$.

$$
n_{1,(z)}^{2}(\lambda)=4.59423+\frac{0.06206}{\lambda^{2}-0.04763}+\frac{110.80672}{\lambda^{2}-86.12171},
$$

where $\lambda$ is the wavelength expressed in $\mu \mathrm{m}$.

The curves in Fig. 2 represent uniform poling-period values satisfying the quasi-phase-matching conditions versus the signal wavelength $\lambda_{s}$, for four different values of the waveguide thickness $h$. The orange (black) curves represent condition 1 (2), namely, that the signal (idler) mode number is 0 and the idler (signal) mode number is 1 . In panels (a) and (b), the interactions are type-0 $(e, e, e)$, with $\left|d_{\mathrm{eff}}\right|=\left|d_{33}\right|$ $=16.9 \mathrm{pm} / \mathrm{V}[21]$. The interactions in panels $(\mathrm{c})$ and (d) are type-II $(o, e, o)$ and type-II $(e, o, o)$, respectively, with $\left|d_{\mathrm{eff}}\right|$ $=\left|d_{24}\right|=3.64 \mathrm{pm} / \mathrm{V}$. The notation $(\cdot, \cdot, \cdot)$ indicates, in consecutive order, the polarization of the down-converted photon whose frequency lies above the degenerate frequency, the down-converted photon whose frequency lies below the degenerate frequency, and the pump photon.

These plots reveal that the two curves can intersect at a single frequency, as in panels (a) and (c) or, alternatively, they can coincide over a broad range of frequencies, as in panels (b) and (d). Modal entanglement can be obtained at any intersection point since the two quasi-phase-matching
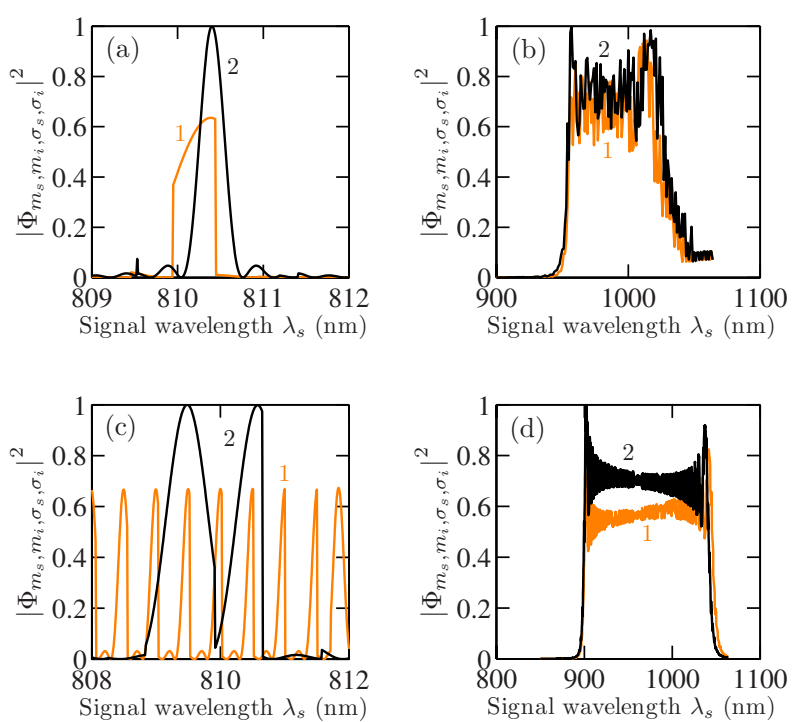

FIG. 3. (Color online) Normalized output spectra $\left|\Phi_{\mathbf{m}, \boldsymbol{\sigma}}\left(\omega_{s}\right)\right|^{2}$ $=\left|\Phi_{m_{s}, m_{i}, \sigma_{s}, \sigma_{i}}\left(\omega_{s}\right) h t\right|^{2}$ as a function of the signal wavelength $\lambda_{s}$, for the four entanglement conditions shown in Fig. 2. Panels (a) and (b) display modal/spectral entanglement regimes, whereas panels (c) and (d) display modal/spectral entanglement and modal/polarization entanglement regimes. The orange (black) curves represent condition 1 (2), namely, that the signal (idler) mode number $m_{s}\left(m_{i}\right)$ is 0 and that the idler (signal) mode number $m_{i}\left(m_{s}\right)$ is 1 . The waveguide length $L$ is $25 \mathrm{~mm}$. (a) Type-0 $(e, e, e)$, uniform poling with $\Lambda_{0}$ $=7.5816 \mu \mathrm{m}$. (b) Type-0 $(e, e, e)$, linearly chirped poling with $\Lambda_{0}$ $=6.87 \mu \mathrm{m}$ and $\Lambda_{L}=6.95 \mu \mathrm{m}$. (c) Type-II $(o, e, o)$, uniform poling with $\Lambda_{0}=19.298 \mu \mathrm{m}$. The multiple resonances that appear in condition 1 result from the rippled behavior of the poling period near the intersection point, as illustrated in the inset of Fig. 2(c). (d) Type-II $(e, o, o)$, linearly chirped poling with $\Lambda_{0}=30 \mu \mathrm{m}$ and $\Lambda_{L}$ $=70 \mu \mathrm{m}$.

conditions are simultaneously satisfied for the unique poling period determined by the point of intersection. In panels (a) and (c), $h$ has been chosen such that the intersection point between the curves occurs near $\lambda_{s}=810 \mathrm{~nm}$ so that the down-converted photon with the longer wavelength lies in the telecommunications window near $1550 \mathrm{~nm}$. In panels (b) and (d), $h$ has been chosen such that the two curves are tangential over a broad range of wavelengths.

We now proceed to study the spectral characteristics for the four structures examined in Fig. 2. The fixed poling periods (spatial frequencies) established for panels (a) and (c) in Fig. 2 are used for calculating the spectra presented in panels (a) and (c) in Fig. 3. Similarly, the range of spatial frequencies where the curves overlap in Figs. 2(b) and 2(d) establishes the linearly chirped spatial frequencies used for the calculations displayed in Figs. 3(b) and 3(d). In all cases, we have chosen a structure of length $L=25 \mathrm{~mm}$.

The output spectra for the two conditions represented by the quantities $\left|\Phi_{0,1, \boldsymbol{\sigma}}\left(\omega_{s}\right)\right|^{2}$ and $\left|\Phi_{1,0, \boldsymbol{\sigma}}\left(\omega_{s}\right)\right|^{2}$ are shown in Fig. 3, normalized to the maximum of their peak values. It suffices to plot these quantities solely for signal frequencies above the degenerate frequency $\omega_{p} / 2$ since 


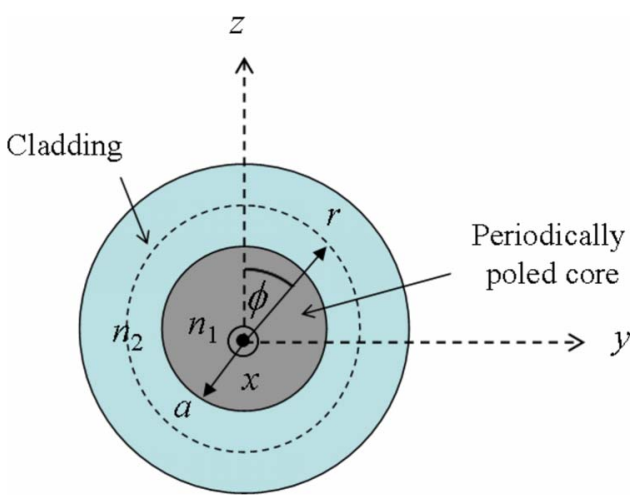

FIG. 4. (Color online) Sketch of the cross section of a 2D circular waveguide. The waveguide is made up of a cylindrical rod of dielectric medium with radius $a$, and uniform refractive index $n_{1}$ surrounded by a medium of lower refractive index $n_{2}$. The inner and the outer media are called the core and the cladding, respectively. The core is periodically poled in the $x$ direction.

$$
\Phi_{0,1, \sigma_{i}, \sigma_{s}}\left(-\omega_{s}\right)=\Phi_{1,0, \sigma_{s}, \sigma_{i}}\left(\omega_{s}\right) .
$$

This relation holds by virtue of the definitions of $A_{\mathbf{m}, \boldsymbol{\sigma}}\left(\omega_{s}\right)$ and $\Delta \beta_{\mathrm{m}, \boldsymbol{\sigma}}\left(\omega_{s}\right)$. Modal entanglement can be achieved over the overlapping regions of the two spectra, as illustrated in Fig. 3. Linearly chirped poling leads to a broadband spectrum by virtue of the continuum of its Fourier domain so that multiple nonlinear interactions can simultaneously be satisfied $[22,23]$. There is, however, a tradeoff between spectral breadth and photon flux density.

The astute reader will have noticed that we have excluded type-I interactions from our considerations. This is because the down-converted photons have the same polarizations as those in type-0, but type-I suffers from lower efficiency. The photon flux from a type- 0 interaction is also expected to be greater than that from a type-II interaction since the former exploits the strongest component of the second-order nonlinear tensor.

\section{MODAL ENTANGLEMENT IN 2D CIRCULAR WAVEGUIDES (SILICA FIBERS)}

Although silica is a centrosymmetric material, secondorder nonlinearities of $\sim 1 \mathrm{pm} / \mathrm{V}$ have been observed in poled silicate fibers (see Fig. 4) [24-27]. The presence of a second-order nonlinearity in this centrosymmetric material appears to stem from the intrinsic third-order nonlinear susceptibility of the glass and the built-in electric field arising from the displacement of charge species created during the poling process. Although the effective nonlinear coefficient $\left|d_{\text {eff }}\right|$ in silica fiber is substantially smaller than that for many second-order nonlinear materials (e.g., $\mathrm{LiNbO}_{3}$ and KTP), silica fibers are nonetheless of interest because they can be fabricated in very long lengths. Second-order interactions in poled glass fibers can be treated using the same techniques as those developed for QPM structures.

In comparison with 1D planar waveguides, which carry the index $m_{q}$, each confined mode of a wave $q$ in a $2 \mathrm{D}$ circular waveguide is marked by an additional index, $l_{q}$.
Based on the geometry of the circular waveguide, cylindrical coordinates are a natural choice for describing the transversemode profile [19],

$$
u_{l_{q} m_{q}}\left(\omega_{q}, r, \phi\right)=\left\{\begin{array}{cc}
J_{l_{q}}\left(k_{l_{q} m_{q}}^{(T)} r\right) \exp \left(j l_{q} \phi\right), & r \leq a \\
K_{l_{q}}\left(\gamma_{l_{q} m_{q}} r\right) \exp \left(j l_{q} \phi\right), & r>a .
\end{array}\right.
$$

Here $r$ and $\phi$ are the radial and azimuthal coordinates, respectively; $l_{q}=0, \pm 1, \pm 2, \ldots ; m_{q}=1,2,3, \ldots ; J_{l_{q}}(\cdot)$ is the Bessel function of the first kind of order $l_{q} ; K_{l}^{q}(\cdot)$ is the modified Bessel function of the second kind of order $l_{q}$; $k_{l_{q} m_{q}}^{(T)}=\left[\left(n_{1} \omega_{q} / c\right)^{2}-\beta_{l_{q} m_{q}}^{2}\right]^{1 / 2} ; \gamma_{l_{q} m_{q}}=\left[\beta_{l_{q} m_{q}}^{2}-\left(n_{2} \omega_{q} / c\right)^{2}\right]^{1 / 2}$; the superscript $(T)$ represents the transverse component of the wave vector $k_{l_{q} m} ; a$ is the radius of the fiber core; and $n_{1}$ and $n_{2}$ are the refractive indexes of the core and cladding, respectively (they are frequency dependent).

The total number of modes $N_{q}$ is determined by the value of the fiber parameter $V_{q}=\left(\omega_{q} a / c\right)\left(n_{1}^{2}-n_{2}^{2}\right)^{1 / 2}$. If, for example, $2.405<V_{q}<3.83$, then $l_{q}=0$, \pm 1 and $m_{q}=1$ so that $N_{q}=3$. For each value of $l_{q}$, the mode propagation constants $\beta_{l_{q} m_{q}}$ can be determined by solving the fiber characteristic equation for $k^{(T)}$ [19],

$$
X \frac{J_{l_{q} \pm 1}(X)}{J_{l_{q}}(X)}= \pm Y \frac{K_{l_{q} \pm 1}(Y)}{K_{l_{q}}(Y)},
$$

where $X=k^{(T)} a$ and $Y=\left(V_{q}^{2}-X^{2}\right)^{1 / 2}$. The values of $k^{(T)}$ that satisfy the characteristic equation are $k_{l_{q} m_{q}}^{(T)}$. Using the definitions provided above, we can then determine $\beta_{l_{q} m_{q}}$. The subscript $\boldsymbol{\sigma}$ has been eliminated in this section since glass fiber is an isotropic material and the characteristic equation is polarization independent.

In $2 \mathrm{D}$ circular waveguides, the two-photon quantum state is

$$
|\Psi\rangle \sim \int d \omega_{s} \sum_{\mathbf{I m}} \Phi_{\mathbf{I m}}\left(\omega_{s}\right)\left|\omega_{s}, l_{s} m_{s}\right\rangle\left|\omega_{i}, l_{i} m_{i}\right\rangle,
$$

where $\mathbf{I m}=\left(l_{s} m_{s}, l_{i} m_{i}\right), \Phi_{\mathbf{I m}}\left(\omega_{s}\right)$ can be determined from Eq. (4) and the amplitude $A_{\operatorname{lm}}\left(\omega_{s}\right)$ in cylindrical coordinates is given by

$$
A_{\operatorname{lm}}\left(\omega_{s}\right)=\int_{0}^{a} d r \int_{0}^{2 \pi} d \phi r \prod_{q=p, s, i} u_{l_{q} m_{q}}\left(\omega_{q}, r, \phi\right) .
$$

Substituting Eq. (17) into Eq. (20) and performing the azimuthal integration leads to $\delta\left(l_{p}+l_{s}+l_{i}\right)$, which can be viewed as a limitation on the transverse profiles of the interacting modes (similar to that for planar waveguides).

Consider nondegenerate SPDC in an optical fiber with $2.405<V_{q}<3.83$. We seek to generate photon pairs with the following entangled state:

$$
\begin{aligned}
|\Psi\rangle \sim & \int d \omega_{s}\left[\Phi_{01,11}\left(\omega_{s}\right)\left|\omega_{s}, 01\right\rangle\left|\omega_{i}, 11\right\rangle+\Phi_{11,01}\left(\omega_{s}\right)\left|\omega_{s}, 11\right\rangle\right. \\
& \left.\times\left|\omega_{i}, 01\right\rangle\right],
\end{aligned}
$$

signifying that if the signal is in the fundamental mode $\left(l_{s} m_{s}=01\right)$, then the idler will be in the first mode $\left(l_{i} m_{i}\right.$ $=11$ ), and vice versa. To have nonvanishing $A_{\operatorname{lm}}\left(\omega_{s}\right)$ or 

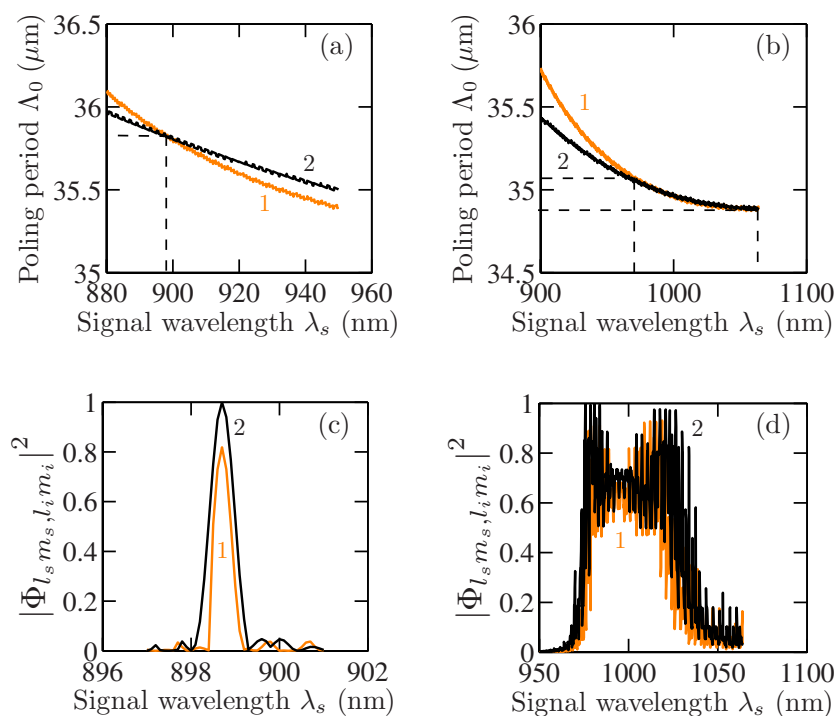

FIG. 5. (Color online) A laser source with wavelength $\lambda_{p}$ $=532 \mathrm{~nm}$ and in mode $l_{p} m_{p}=-11$ is used to pump a poled silica fiber with $\Delta=0.01$. [(a) and (b)] Poling period versus the signal wavelength $\lambda_{s}$, with core radii $a=2.613$ and $a=2.52 \mu \mathrm{m}$, respectively. The orange (black) curve represents condition 1 (2), namely, that the signal (idler) mode number $l_{s} m_{s}\left(l_{i} m_{i}\right)$ is 01 and the idler (signal) mode number $l_{i} m_{i}\left(l_{s} m_{s}\right)$ is 11 . Dashed lines show the intersection point or region. [(c) and (d)] These panels display modal/ spectral entanglement regimes. Normalized output spectra $\left|\Phi_{01,11}\left(\omega_{s}\right)\right|^{2}$ and $\left|\Phi_{11,01}\left(\omega_{s}\right)\right|^{2}$ for the structures displayed in (a) and (b), respectively, with $L=20 \mathrm{~cm}$. The poling period of the nonlinear coefficient is uniform in (c) with $\Lambda_{0}=35.813 \mu \mathrm{m}$, and is linearly chirped in (d) with $\Lambda_{0}=34.9 \mu \mathrm{m}$ and $\Lambda_{L}=35.05 \mu \mathrm{m}$.

$\Phi_{\operatorname{lm}}\left(\omega_{s}\right)$, the pump mode index $l_{p} m_{p}$ must be $(-11)$.

Example. A pump source, with wavelength $\lambda_{p}=532 \mathrm{~nm}$ and in mode $l_{p} m_{p}=-11$, is incident on an optical glass poled fiber with fractional refractive-index change $\Delta=0.01$. The core refractive index is computed using the Sellmeier equation for fused silica [19],

$$
\begin{aligned}
n_{1}^{2}(\lambda)= & 1+\frac{0.6962 \lambda^{2}}{\lambda^{2}-(0.06840)^{2}}+\frac{0.4079 \lambda^{2}}{\lambda^{2}-(0.1162)^{2}} \\
& +\frac{0.8975 \lambda^{2}}{\lambda^{2}-(9.8962)^{2}},
\end{aligned}
$$

where $\lambda$ is the wavelength expressed in $\mu \mathrm{m}$. Panels (a) and (b) in Fig. 5 represent the dependence of the uniform poling period on the signal wavelength $\lambda_{s}$ required to satisfy quasiphase-matching for the two conditions under consideration, for two different values of the core radius $a$. The curves can either intersect at a single frequency or they can coincide over a broad range of frequencies [in analogy with the results for the 1D planar waveguide; see Figs. 2(a) and 2(b)]. Modal entanglement can be achieved where the curves intersect. The core radius $a$ for panel (a) has been chosen such that the intersection occurs at $\lambda_{s}=900 \mathrm{~nm}$, placing the downconverted photon with the longer wavelength in the telecommunications window near $1300 \mathrm{~nm}$. In panel (b), $a$ has been selected such that the two curves are tangential over a broad band of frequencies. Panels (c) and (d) in Fig. 5 display the normalized output spectra $\left|\Phi_{01,11}\left(\omega_{s}\right)\right|^{2}$ and $\left|\Phi_{11,01}\left(\omega_{s}\right)\right|^{2}$ for fibers of length $L=20 \mathrm{~cm}$. The poling periods of the fibers used in (c) and (d) are determined from the results obtained in (a) and (b), respectively. Modal entanglement can be achieved in wavelength regions where the spectra overlap.

\section{FEATURES OF MODAL ENTANGLEMENT}

In the previous sections, we have demonstrated that it is possible to obtain modal entanglement in 1D planar and 2D circular waveguides. In this section, we discuss various features and applications of modal entanglement.

\section{A. Combining the advantages of noncollinear and collinear-degenerate interactions in bulk crystals}

A noncollinear configuration is often preferred in bulk crystals because the photon pairs generated by SPDC are easily separated. This process is typically not efficient, however, since the fraction of the photons that are entangled is seriously limited by the intersections between the emission cones. A degenerate-collinear configuration, on the other hand, creates impediments to separating the photon pairs but offers high efficiency by virtue of the total overlap of the emission cones. In a two-mode waveguide with modal entanglement, in contrast, the photon pairs are efficiently generated in the two allowed entangled modes and are, thus, also readily separated by their mode numbers, i.e., their different transverse-field profiles. This can be implemented by use of a branching waveguide [28].

\section{B. Device flexibility and compatibility}

Using uniform or linearly chirped poling, we are able to generate either narrowband or broadband spectral/ polarization entanglement, as illustrated in Fig. 3. By replacing the branching waveguide at the device output with a diffraction grating, prism, or polarizing beam splitter (for type-II), we can obtain binary modal entanglement. The structure can then be used as a source of binary and continuum entanglement that is expected to be useful for applications such as quantum imaging [29-31], quantum cryptography [32], quantum teleportation [33], and quantum information [34]. We note that this waveguide configuration is compatible with integrated optics, which can facilitate its incorporation into a practical system.

\section{Spectral, polarization, and modal entanglement}

Spectral entanglement is typically measured via a HongOu-Mandel interferometer (HOM) [35]. An experiment is conducted by sweeping a temporal delay $\tau$ inserted between the down-converted photons, while measuring the coincidence rate of photon counts at a pair of detectors placed at the two output ports of the interferometer. The coincidence rate $R(\tau)$ is given by

$$
R(\tau)=\frac{1}{2}\left\{R_{0}-\operatorname{Re}\left[R_{1}(\tau)\right]\right\}
$$

with 

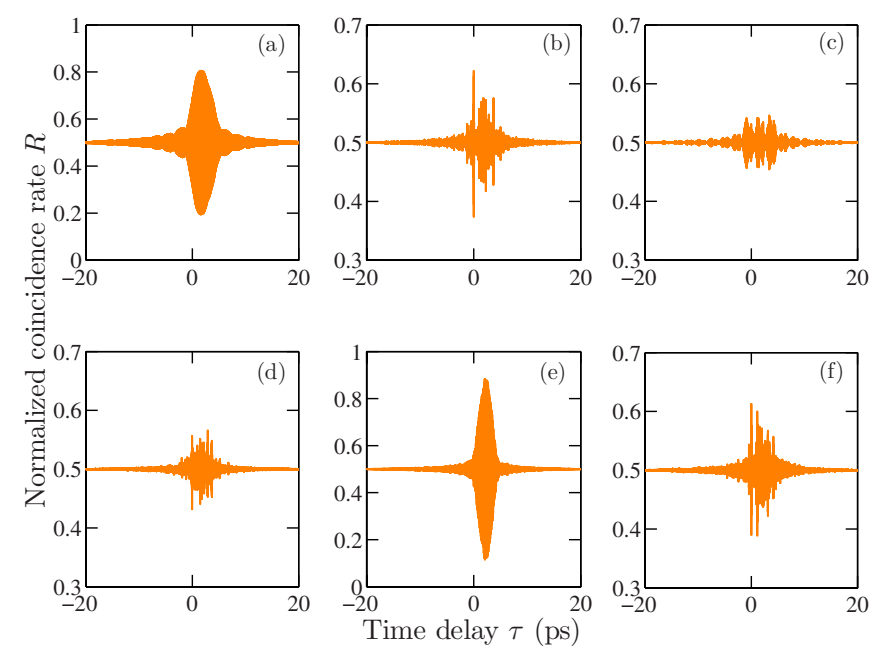

FIG. 6. (Color online) Dependence of the normalized coincidence rate $R$ on the time delay $\tau$ between the two photons calculated for a HOM interferometer. (a) 1D planar waveguide with uniform poling and type-0 interaction. (b) $1 \mathrm{D}$ planar waveguide with linearly chirped poling and type- 0 interaction. (c) $1 \mathrm{D}$ planar waveguide with uniform poling and type-II interaction. (d) 1D planar waveguide with linearly chirped poling and type-II interaction. (e) 2D circular waveguide with uniform poling and type-0 interaction. (f) $2 \mathrm{D}$ circular waveguide with linearly chirped poling and type- 0 interaction. The structure and operational parameters for panels (a), (b), (c), (d), (e), and (f) are the same as those used for Figs. 3(a)-3(d), 5(c), and 5(d), respectively.

$$
\begin{gathered}
R_{0}=\int d \omega_{s}\left|\Phi_{\mathbf{m}, \boldsymbol{\sigma}}\left(\omega_{s}\right)\right|^{2}, \\
R_{1}(\tau)=\int d \omega_{s}\left\{\Phi_{\mathbf{m}, \boldsymbol{\sigma}}\left(\omega_{s}\right) \Phi_{\mathbf{m}, \boldsymbol{\sigma}}^{*}\left(-\omega_{s}\right) \exp \left[j\left(\omega_{p}-2 \omega_{s}\right) \tau\right]\right\},
\end{gathered}
$$

where $\mathrm{Re}$ denotes the real part and the superscript * represents the complex conjugate. The integration covers the entire spectrum so that either $\Phi_{m_{0}, m_{1}, \boldsymbol{\sigma}}\left(\omega_{s}\right)$ or $\Phi_{m_{1}, m_{0}, \boldsymbol{\sigma}}\left(\omega_{s}\right)$ can be used to evaluate the integral, where $m_{0}$ and $m_{1}$ are the two lowest modes of a waveguide. Again, changing the spatialmode number is equivalent to changing the sign of the frequency, i.e., $\Phi_{m_{0}, m_{1}, \boldsymbol{\sigma}}\left(-\omega_{s}\right)=\Phi_{m_{1}, m_{0}, \boldsymbol{\sigma}}\left(\omega_{s}\right)$.

The graphs presented in Fig. 6 are the normalized coincidence rate expected for an HOM interferometer for the planar and circular waveguide structures described in Secs. III and IV, respectively. Since the signal and idler are nondegenerate, the familiar dip in the coincidence interferogram is modulated by an oscillatory function. When using the mode numbers for identifying the photons, two different phasematching conditions are required to obtain spectral or polarization entanglement in waveguides, rather than just a single one for bulk crystals. This distinction arises because of the dependence of the propagation constant on the mode number in a waveguide; in bulk crystals the magnitude of the wave vector is independent of the direction of propagation. Hence, $\Delta \widetilde{\beta}_{m_{0}, m_{1}, \boldsymbol{\sigma}}\left(\omega_{s}\right)$ is not exactly equal to $\Delta \widetilde{\beta}_{m_{0}, m_{1}, \boldsymbol{\sigma}}\left(-\omega_{s}\right)$, so that
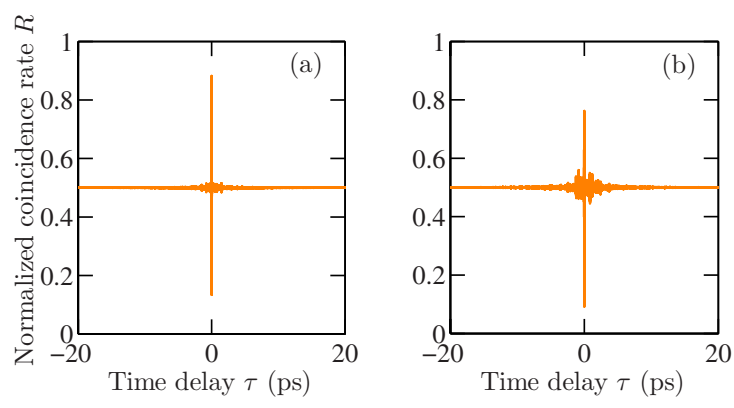

FIG. 7. (Color online) Dependence of the normalized coincidence rate $R$ on the time delay $\tau$ between the two photons for a HOM interferometer. (a) 1D planar waveguide with linearly chirped poling and type-II interaction. (b) 2D circular waveguide with linearly chirped poling and type-0 interaction. The structure and operational parameters for panel (a) are the same as those used for Figs. 3(d) and 6(d) except that $L=2 \mathrm{~mm}$ instead of $25 \mathrm{~mm}$. The structure and the operational parameters for panel (b) are the same as those used for Figs. 5(d) and 6(f) except that $L=20 \mathrm{~mm}$ instead of $20 \mathrm{~cm}$.

$\Phi_{\mathbf{m}, \boldsymbol{\sigma}}\left(\omega_{s}\right)$ and $\Phi_{\mathbf{m}, \boldsymbol{\sigma}}^{*}\left(-\omega_{s}\right)$ do not perfectly overlap in the complex domain. This has several consequences: (i) The interference pattern shifts about $\tau=0$ since $\Phi_{\mathbf{m}, \boldsymbol{\sigma}}\left(\omega_{s}\right) \Phi_{\mathbf{m}, \boldsymbol{\sigma}}^{*}$ $\times\left(-\omega_{s}\right)$ is a not purely real. This effect is illustrated in Figs. 6(a) and 6(e). (ii) The visibility, which is defined as $\tilde{V}(\tau)$ $=\left(R_{\max }-R_{\min }\right) /\left(R_{\max }+R_{\min }\right)$, where $R_{\max }$ and $R_{\min }$ are the maximum and minimum values of $R(\tau)$, respectively, is reduced since $R_{0}$ is not precisely equal to $R_{\min }$. This is clearly observable in the type-II curves portrayed in Figs. 6(c) and 6(d). (iii) The interference pattern is ragged, as depicted in Figs. 6(b) and 6(f). However, reducing the device length will result in a cleaner interference pattern since the imaginary part of $\Phi_{\mathbf{m}, \boldsymbol{\sigma}}\left(\omega_{s}\right) \Phi_{\mathbf{m}, \boldsymbol{\sigma}}^{*}\left(-\omega_{s}\right)$ is proportional to the device length for both uniform and linearly chirped poling.

Using Eqs. (8) and (10), we define the entanglement length $L_{e}$ as the waveguide length for which the phase of $\Phi_{\mathbf{m}, \boldsymbol{\sigma}}\left(\omega_{s}\right) \Phi_{\mathbf{m}, \boldsymbol{\sigma}}^{*}\left(-\omega_{s}\right)$ is equal to $\pi$. For uniform poling, we obtain

$$
L_{e}=\left|\frac{2 \pi}{\Delta \widetilde{\beta}_{m_{0}, m_{1}, \boldsymbol{\sigma}}\left(\omega_{s}\right)-\Delta \widetilde{\beta}_{m_{0}, m_{1}, \boldsymbol{\sigma}}\left(-\omega_{s}\right)}\right|,
$$

whereas for linearly chirped poling, we have

$$
L_{e}=\left|\frac{4 \pi^{2}\left(\Lambda_{0}^{-1}-\Lambda_{L}^{-1}\right)}{\Delta \widetilde{\beta}_{m_{0}, m_{1}, \boldsymbol{\sigma}}^{2}\left(\omega_{s}\right)-\Delta \widetilde{\beta}_{m_{0}, m_{1}, \boldsymbol{\sigma}}^{2}\left(-\omega_{s}\right)}\right| .
$$

To explicitly illustrate that the character of the interference pattern does depend on the device length, we present plots of the coincidence rate for shorter structures in Fig. 7. The visibility of the interference pattern increases and its "noisiness" decreases.

Polarization entanglement is measured by splitting a pair of photons using a nonpolarizing beam splitter and then sending them to two Glan-Thompson analyzers [36]. After passage through analyzers oriented at angles $\theta_{1}$ and $\theta_{2}$, the coincidence rate turns out to be 

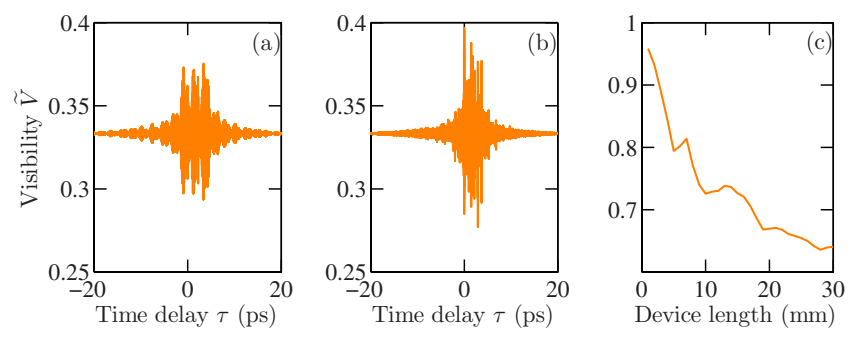

FIG. 8. (Color online) [(a) and (b)] Dependence of the visibility $\tilde{V}$ of the interference pattern on the time delay $\tau$ between the downconverted photons in type-II interactions for $1 \mathrm{D}$ planar waveguides with uniform and linearly chirped poling, respectively. The structure and operational parameters for panels (a) and (b) are the same as those used for Fig. 3(c) and 3(d), respectively. (c) Dependence of the visibility $\widetilde{V}$ on the device length $L$ for a $1 \mathrm{D}$ planar waveguide with uniform poling in a type-II interaction. The parameters are the same as those used for Fig. 2(d), with a uniform poling period $\Lambda_{0}$ $=67.7768 \mu \mathrm{m}$

$$
R\left(\theta_{1}, \tau\right)=\frac{1}{4}\left(R_{0}-\frac{1}{2} \sin ^{2}\left(2 \theta_{1}\right)\left\{R_{0}+\operatorname{Re}\left[R_{1}(\tau)\right]\right\}\right),
$$

where $\theta_{1}+\theta_{2}=90^{\circ}$; the angles are measured with respect to the $o$-polarization wave. The visibility of the interference pattern is given by $\tilde{V}(\tau)=\left\{R_{0}+\operatorname{Re}\left[R_{1}(\tau)\right]\right\} /\left\{3 R_{0}-\operatorname{Re}\left[R_{1}(\tau)\right]\right\}$. The dependence of the visibility on temporal delay is displayed in Figs. 8(a) and 8(b) for structures that support type-II interactions. The effect of the waveguide length on the visibility is depicted in Fig. 8(c), which displays calculations carried out for a 1D KTP planar waveguide pumped using an $o$-polarized wave to generate type-II $(e, o, o)$ downconversion. The nonlinear coefficient is taken to be uniformly poled with a period $\Lambda_{0}=67.7768 \mu \mathrm{m}$ so that the wavelength of one of the down-converted photons lies in one of the optical telecommunications windows. The other parameters are the same as those used for Fig. 2(d). The visibility is seen to degrade with increasing waveguide length. Hence, there is a tradeoff between long waveguide length to generate a high flux of photon pairs and short waveguide length to generate a smooth interference pattern with high visibility.

Modal entanglement can be considered as an alternative to polarization entanglement. Whereas polarization entanglement is restricted to type-II interactions, modal entanglement has the merit that it can be generated using any type of nonlinear interaction, including type-0. Indeed, the downconversion generation rate in type- 0 is about one order of magnitude higher than that in type-II since type-0 utilizes the strongest component of the second-order nonlinear tensor. Hence, SPDC generated via type- 0 modal entanglement in a waveguide can serve as an efficient source of binary entangled photons.

\section{Improvement in the overall signal-to-noise ratio of an infrared biphoton system}

An increase in the overall signal-to-noise ratio in an infrared biphoton system can be achieved by choosing the waveguide dimensions such that one of the photons falls in
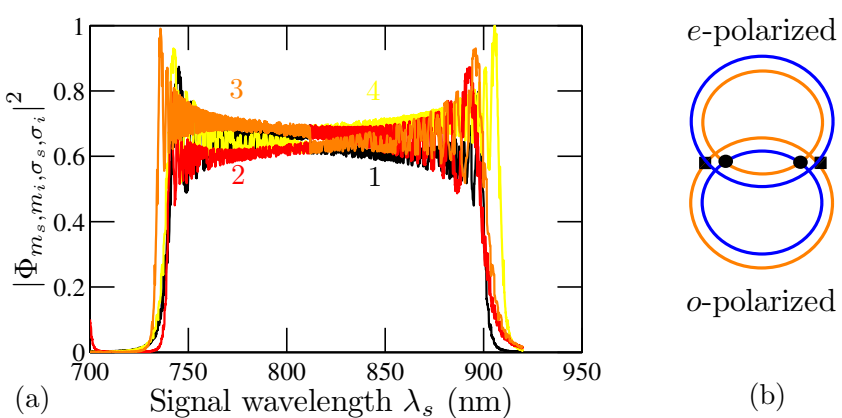

(b)

FIG. 9. (Color online) (a) Output spectra of SPDC from a KTP planar waveguide with thickness $h=1.1 \mu \mathrm{m}$, length $L=25 \mathrm{~mm}$, and $\Delta=0.05$, using an $o$-polarized source in mode $m_{p}=1$ at $\lambda_{p}$ $=406 \mathrm{~nm}$. The poling period of the nonlinear coefficient is linearly chirped with $\Lambda_{0}=6.5 \mu \mathrm{m}$ and $\Lambda_{L}=7.9 \mu \mathrm{m}$. The black (1) and red (2) curves represent the output spectra associated with type-II $(o, e, o)$ modal entanglement, while the orange (3) and yellow (4) curves represent the output spectra associated with type-II $(e, o, o)$ modal entanglement. The regions where the four spectra overlap indicate modal/spectral/polarization entanglement. (b) The regions of bulk type-II $(o, e, o)$ and type-II $(e, o, o)$ polarization entanglement are marked by small black filled circles and squares, respectively. The blue and orange rings represent photons with frequencies above and below the degenerate frequency, respectively.

the visible region while the other lies in the infrared region. This technique can be useful since the visible photon can be efficiently detected with a Si photon-counting detector, while the infrared photon is detected with a less efficient InGaAs photon-counting detector. The overall signal-to-noise ratio is increased as a consequence [30,37].

\section{E. Generation of a doubly entangled state via modal entanglement}

Although type-II is less efficient than type-0, this configuration can be exploited to generate a doubly entangled state in frequency and polarization [8],

$$
|\Psi\rangle \sim\left(\left|\omega_{s}, \omega_{i}\right\rangle+\left|\omega_{i}, \omega_{s}\right\rangle \otimes\left|\sigma_{s}, \sigma_{i}\right\rangle+\left|\sigma_{i}, \sigma_{s}\right\rangle\right) .
$$

This state can be viewed as a combination of type-II $(o, e, o)$ and type-II $(e, o, o)$. The photon pairs can be separated on the basis of their mode number with the help of a branching waveguide. Using Eq. (6), four different nonlinear processes are required to generate the doubly-entangled state,

$$
\begin{aligned}
|\Psi\rangle \sim & \int d \omega_{s}\left[\Phi_{0,1, o, e}\left(\omega_{s}\right)\left|\omega_{s}, o\right\rangle_{0}\left|\omega_{i}, e\right\rangle_{1}+\Phi_{1,0, o, e}\left(\omega_{s}\right)\right. \\
& \times\left|\omega_{i}, e\right\rangle_{0}\left|\omega_{s}, o\right\rangle_{1}+\Phi_{0,1, e, o}\left(\omega_{s}\right)\left|\omega_{s}, e\right\rangle_{0}\left|\omega_{i}, o\right\rangle_{1} \\
& \left.+\Phi_{1,0, e, o}\left(\omega_{s}\right)\left|\omega_{i}, o\right\rangle_{0}\left|\omega_{s}, e\right\rangle\right]_{1} .
\end{aligned}
$$

Although it is difficult to satisfy the phase-matching condition for all of these processes using uniform poling, this can be achieved by using linearly chirped poling. The nonlinear coefficient can also be poled in an aperiodic sequence to obtain a narrowband doubly-entangled state [38].

In Fig. 9(a), we plot the normalized output spectra $\left|\Phi_{m_{s}, m_{i}, \sigma_{s}, \sigma_{i}}\right|^{2}$ when an $o$-polarized pump source in mode $m_{p}$ 
$=1$ at $\lambda_{p}=406 \mathrm{~nm}$ is incident on a 1D KTP planar waveguide with thickness $h=1.1 \mu \mathrm{m}$, length $L=25 \mathrm{~mm}$, and $\Delta$ $=0.05$. The poling period of the nonlinear coefficient is linearly chirped with $\Lambda_{0}=6.5 \mu \mathrm{m}$ and $\Lambda_{L}=7.9 \mu \mathrm{m}$. As illustrated in Fig. 9, the output spectra associated with type-II $(o, e, o)$ modal entanglement overlap with the output spectra associated with type-II $(e, o, o)$ modal entanglement. The region of overlap between the four spectra (except for the degenerate case) represents the spectral band over which a doubly-entangled state can be obtained. It is worthy of note that this state cannot be generated in bulk crystals since four different directions are required, as shown in Fig. 9(b). In waveguides with modal entanglement, however, only two mode numbers are required.

\section{CONCLUSION}

We have investigated nondegenerate spontaneous parametric down-conversion (SPDC) in multimode 1D planar and 2D circular waveguides. Using various types of interactions (type-0 or II), the generated photons can be entangled in the two lowest mode numbers of the waveguide-we refer to this as "modal entanglement." The inherent phase mismatch in the process is corrected by modulating the nonlinear coefficient with poling periods (spatial frequencies) that are either uniform or linearly chirped. Since the interaction is collinear within the waveguides, modal entanglement can be used in place of directional entanglement to distinguish be- tween the down-converted photons. Spectral or polarization entanglement can, therefore, be generated with high efficiency and the entangled photons can be readily separated. We find that there is a tradeoff between long waveguide length to generate a high flux of photon pairs and short waveguide length to generate a smooth interference pattern with high visibility. In type- 0 interactions, frequency can be used as a mode identifier rather than waveguide mode number, offering the possibility of using modal entanglement in place of polarization entanglement, which requires a type-II interaction. This is a salutary feature since a type- 0 interaction exploits the strongest component of the second-order nonlinear tensor. The technique can be implemented in any of the optical telecommunications windows by controlling the waveguide dimensions. Finally, we have demonstrated that modal entanglement in a type-II interaction can be used to generate a doubly-entangled state in frequency and polarization. Drawing on a Hilbert space of higher dimensions can offer advantages in quantum-communication protocols and quantum computing.

\section{ACKNOWLEDGMENTS}

This work was supported by a U.S. Army Research Office (ARO) Multidisciplinary University Research Initiative (MURI) Grant and by the Bernard M. Gordon Center for Subsurface Sensing and Imaging Systems (CenSSIS), an NSF Engineering Research Center.
[1] D. N. Klyshko, Photons and Nonlinear Optics (Nauka, Moscow, 1980), Chaps. 1 and 6.

[2] B. E. A. Saleh, A. F. Abouraddy, A. V. Sergienko, and M. C. Teich, Phys. Rev. A 62, 043816 (2000).

[3] A. Joobeur, B. E. A. Saleh, and M. C. Teich, Phys. Rev. A 50, 3349 (1994).

[4] A. Joobeur, B. E. A. Saleh, T. S. Larchuk, and M. C. Teich, Phys. Rev. A 53, 4360 (1996).

[5] A. Mair, A. Vaziri, G. Weihs, and A. Zeilinger, Nature (London) 412, 313 (2001).

[6] A. F. Abouraddy, T. Yarnall, B. E. A. Saleh, and M. C. Teich, Phys. Rev. A 75, 052114 (2007).

[7] T. Yarnall, A. F. Abouraddy, B. E. A. Saleh, and M. C. Teich, Phys. Rev. Lett. 99, 170408 (2007).

[8] J. T. Barreiro, N. K. Langford, N. A. Peters, and P. G. Kwiat, Phys. Rev. Lett. 95, 260501 (2005).

[9] M. Halder, S. Tanzilli, H. de Riedmatten, A. Beveratos, H. Zbinden, and N. Gisin, Phys. Rev. A 71, 042335 (2005).

[10] J. G. Rarity and P. R. Tapster, Phys. Rev. Lett. 64, 2495 (1990).

[11] S. P. Walborn, S. Pádua, and C. H. Monken, Phys. Rev. A 71, 053812 (2005).

[12] M. C. Booth, M. Atature, G. DiGiuseppe, B. E. A. Saleh, A. Sergienko, and M. C. Teich, Phys. Rev. A 66, 023815 (2002).

[13] P. G. Kwiat, K. Mattle, H. Weinfurter, A. Zeilinger, A. V. Sergienko, and Y. Shih, Phys. Rev. Lett. 75, 4337 (1995).

[14] T. Yarnall, A. F. Abouraddy, B. E. A. Saleh, and M. C. Teich,
Phys. Rev. Lett. 99, 250502 (2007)

[15] C. H. Bennett and P. W. Shor, IEEE Trans. Inf. Theory 44, 2724 (1998).

[16] K. Banaszek A. B. U'Ren, and I. A. Walmsley, Opt. Lett. 26, 1367 (2001).

[17] A. Eckstein and C. Silberhorn, Opt. Lett. 33, 1825 (2008).

[18] G. Imeshev, M. A. Arbore, M. M. Fejer, A. Galvanauskas, M. Fermann, and D. Harter, J. Opt. Soc. Am. B 17, 304 (2000).

[19] B. E. A. Saleh and M. C. Teich, Fundamentals of Photonics, 2nd ed. (Wiley, Hoboken, NJ, 2007).

[20] K. Kato and E. Takaoka, Appl. Opt. 41, 5040 (2002).

[21] R. L. Sutherland, Handbook of Nonlinear Optics, 2nd ed. (CRC, Boca Raton, FL, 2003).

[22] S. Carrasco, J. P. Torres, L. Torner, A. V. Sergienko, B. E. A. Saleh, and M. C. Teich, Opt. Lett. 29, 2429 (2004).

[23] M. B. Nasr, S. Carrasco, B. E. A. Saleh, A. V. Sergienko, M. C. Teich, J. P. Torres, L. Torner, D. S. Hum, and M. M. Fejer, Phys. Rev. Lett. 100, 183601 (2008).

[24] H. Nasu, H. Okamoto, K. Kurachi, J. Matsuoka, K. Kamiya, A. Mito, and H. Hosono, J. Opt. Soc. Am. B 12, 644 (1995).

[25] P. G. Kazansky, P. S. J. Russell, and H. Takebe, J. Lightwave Technol. 15, 1484 (1997).

[26] P. G. Kazansky and V. Pruneri, J. Opt. Soc. Am. B 14, 3170 (1997).

[27] J. Fage-Pedersen, R. Jacobsen, and M. Kristensen, Opt. Express 13, 8514 (2005).

[28] H. Yajima, Appl. Phys. Lett. 22, 647 (1973). 
[29] A. F. Abouraddy, B. E. A. Saleh, A. V. Sergienko, and M. C. Teich, Phys. Rev. Lett. 87, 123602 (2001).

[30] A. F. Abouraddy, B. E. A. Saleh, A. V. Sergienko, and M. C. Teich, J. Opt. Soc. Am. B 19, 1174 (2002).

[31] M. B. Nasr, D. P. Goode, N. Nguyen, G. Rong, L. Yang, B. M. Reinhard, B. E. A. Saleh, and M. C. Teich, Opt. Commun. 282, 1154 (2009).

[32] T. Jennewein, C. Simon, G. Weihs, H. Weinfurter, and A. Zeilinger, Phys. Rev. Lett. 84, 4729 (2000).

[33] C. H. Bennett, G. Brassard, C. Crépeau, R. Jozsa, A. Peres, and W. K. Wootters, Phys. Rev. Lett. 70, 1895 (1993).

[34] M. A. Nielsen and I. L. Chuang, Quantum Computation and Quantum Information (Cambridge University Press, Cambridge, UK, 2000).

[35] C. K. Hong, Z. Y. Ou, and L. Mandel, Phys. Rev. Lett. 59, 2044 (1987)

[36] Y. H. Shih and C. O. Alley, Phys. Rev. Lett. 61, 2921 (1988).

[37] H. Guillet de Chatellus, A. V. Sergienko, B. E. A. Saleh, M. C. Teich, and G. Di Giuseppe, Opt. Express 14, 10060 (2006).

[38] A. Norton and C. de Sterke, Opt. Express 12, 841 (2004). 